\title{
Klebsiella pneumoniae Infection: A Virulent Cause of Visual Loss
}

\author{
Wai Cheong Soon Anna Pouncey Elizabeth Ashley \\ Elizabeth Frances Bowen \\ Charing Cross Hospital, London, UK
}

Key Words

Klebsiella pneumoniae $\cdot$ Bacterial endophthalmitis · Visual loss

\begin{abstract}
Bacterial endophthalmitis is endogenous in $2-6 \%$ of cases and is frequently misdiagnosed initially. Klebsiella pneumoniae is being increasingly recognised as an aggressive causative organism, and it is particularly prevalent in Asian populations. We describe the case of a 71year-old female of Southeast Asian origin with type 2 diabetes mellitus who presented with visual loss secondary to bacterial endophthalmitis and concomitant cerebral abscesses. Imaging revealed the probable primary source of infection to be a liver abscess. She developed retinal detachment and subsequently underwent an evisceration of her right eye. A Klebsiella spp. was identified from the eye tissue by $16 \mathrm{~S}$ rRNA amplification. Klebsiella pneumoniae endophthalmitis has a characteristic disease phenotype and a particularly aggressive course with poor visual outcomes observed in most cases. This case highlights the risks of metastatic infection including bacterial endophthalmitis in association with Klebsiella infection.
\end{abstract}

(C) 2014 S. Karger AG, Basel

\section{Introduction}

Endophthalmitis, or infection of the vitreous or aqueous humour or both, is a medical emergency. The average annual incidence of endogenous bacterial endophthalmitis is estimated to be 5 per 10,000 hospitalised patients [1], and Klebsiella pneumoniae is being increasingly recognised as a causative organism. It is particularly prevalent amongst Asian populations [2-4], and its association with diabetes mellitus has been well reported. $K$. pneumoniae endophthalmitis typically occurs as a complication of a primary hepatic infec- 
tion $[5,6]$. It has a characteristic disease phenotype and a particularly aggressive course, with poor visual outcomes observed in $89 \%$ of cases [5].

We have found 1 other case report of Klebsiella endophthalmitis as an initial presentation of metastatic infection from a liver abscess in the UK [7].

\section{Case Presentation}

A 71-year-old female, born in Thailand, who had lived in the UK for more than 20 years, presented to an eye casualty department with a 10-day history of intermittent fever and rigors, accompanied by awareness of floaters and pain in the right eye. She had a background of diet-controlled diabetes, and she had been treated for a presumptive urinary tract infection by her general practitioner 4 days prior to presentation with oral co-amoxiclav.

An initial ophthalmic examination of her right eye revealed a severe anterior uveitis and a hypopyon, accompanied by keratic precipitates and fibrin deposition. Punctate corneal epithelial staining and injected conjunctiva were also noted, and the retina could not be visualised. This was accompanied by increasing visual loss.

Her right eye was almost completely insensitive to light, although direct and consensual pupillary reflexes were preserved. Visual acuity and fields in her left eye were normal, and there were no signs of infection or inflammation. The intraocular pressure in the right and left eye was 32 and $10 \mathrm{~mm} \mathrm{Hg}$, respectively. B-scan ultrasonography revealed a retinal traction over the posterior pole, but no haemorrhage or choroidal masses were noted. The patient was started on cyclopentolate $1 \%$ eyedrops, betamethasone and neomycin (Betnesol$\mathrm{n}$ ) eye ointment, and dexamethasone $0.1 \%$ eyedrops. Her clinical examination was unremarkable; however, blood tests revealed raised inflammatory markers with a white blood cell count of $14.7 \times 10^{9} / \mathrm{l}$ and C-reactive protein of $128 \mathrm{mg} / \mathrm{l}$.

An unenhanced CT of her brain on the day of her initial assessment had shown no focal abnormalities, but a contrast-enhanced CT of her orbits 3 days later demonstrated rightsided proptosis with preseptal and intraconal retro-ocular soft tissue stranding and thickening as well as evidence of associated episcleritis (fig. 1). The patient was commenced on topical dexamethasone, ofloxacin and dorzolamide. Following a transient episode of speech arrest, the patient was admitted to the stroke unit of another hospital and was found to have a temperature of $38^{\circ} \mathrm{C}$. An MRI of her head showed multiple foci of tiny peripherally enhancing lesions with a slightly T2 hypointense wall, surrounding oedema, and a restricted core on diffusion-weighted imaging. Appearances were in keeping with multiple abscesses, most likely pyogenic in nature (fig. 2). Due to the patient's clinical condition, she was not transferred back to the eye hospital for further management of her endophthalmitis and did not receive an intravitreal injection of antibiotics. No primary source of infection was evident on clinical examination, and the patient was empirically commenced on intravenous ceftriaxone and metronidazole to likely target the bacterial causes of her brain abscesses.

Five sets of blood cultures and a culture of 3 eye swabs failed to grow a pathogen and an HIV antibody screen, and the culture of methicillin-resistant Staphylococcus aureus screening swabs were both negative. A transthoracic echocardiogram showed a thickened mitral valve with no vegetation. A liver ultrasound scan revealed a hepatic abscess (not amenable to drainage) which was characterised further with CT. This confirmed a likely biliary source of sepsis secondary to calculous cholecystitis and obstruction of the common bile duct. The patient had no abdominal symptoms at that time and only had mildly deranged liver function tests (bilirubin $36 \mu \mathrm{mol} / \mathrm{l}$, alanine transaminase $61 \mathrm{IU} / \mathrm{l}$ and alkaline phosphatase 140 IU/l). An endoscopic retrograde cholangiopancreatography (ERCP) was attempted but 
Soon et al.: Klebsiella pneumoniae Infection: A Virulent Cause of Visual Loss

abandoned due to technical difficulties. Our patient's blood glucose level on admission was $12.3 \mathrm{mmol} / \mathrm{l}$. She was known to be homozygous for $\mathrm{HbE}$ and did not produce $\mathrm{HbAO}$ and HbA1c; therefore, we were unable to assess her prior glycaemic control.

A week later, the patient had no improvement in vision in her right eye despite being on topical antibiotics, steroids and intravenous antibiotics. However, she was generally feeling better and her inflammatory markers were improving. A slit-lamp examination of the right eye revealed a hazy, opacified cornea, $360^{\circ}$ iridocorneal touch, and $4+$ white blood cells in the anterior chamber. B-scan ultrasonography of the right eye showed a thickened sclera and retinal detachment. The dense cataract in the right eye caused the fundus view to be obscured. A relative afferent pupillary defect was noted in the right eye, and there was no perception to light in the right eye.

A repeat MRI showed a progression in her perilesional oedema as well as a slight growth of the ring-enhancing lesions (fig. 3). CT orbits demonstrated extensive abnormalities around the right globe, retinal detachments and retro-orbital chemosis. The patient was commenced on Pred Forte 1, atropine 1, dorzolamide 2, Betnesol ointment, cyclopentolate 1 and polyvinyl alcohol $1.4 \%$ eyedrops. Intravenous ceftriaxone and metronidazole were continued.

Unfortunately, she went on to develop a perforation in her right cornea, which necessitated the evisceration of her right eye in the following week. This procedure was uncomplicated. Her eye tissue was sent for $16 \mathrm{~S}$ ribotyping, and Klebsiella was identified to species level.

A gradual clinical improvement after her intravenous antibiotic therapy was observed in our patient, with a concurrent reduction in inflammatory markers. She was discharged on an outpatient antibiotic therapy, which was continued for a total of 5 weeks of antibiotics. She was also started on metformin and gliclazide for her diabetes. A repeat MRI scan at 5 months displayed a complete resolution of all ring-enhancing lesions. The patient made a good clinical recovery and returned to living independently with monocular vision. Her left eye remained unaffected and healthy when reviewed at the outpatient clinic 6 months following her discharge. She had a repeat ERCP, and cholecystectomy was recommended.

We would like to thank our patient for consenting to this report of her illness and her clinical course.

\section{Discussion}

The majority of cases of bacterial endophthalmitis are exogenous, occurring after a trauma or surgery. Endogenous endophthalmitis, resulting from haematogenous spread of an infection, is much less common (2-15\% of all cases). Examples of typical sources of infection include endocarditis, complicated urinary tract infections, line-related infections and liver abscesses. Endophthalmitis has occasionally been described in association with a transient bacteraemia, e.g. in injection drug users or following endoscopy [8].

Numerous organisms have been implicated with $S$. aureus and Streptococcus spp. being among the most common. Only 5 and $3.6 \%$ of cases were attributable to K. pneumoniae infections in the UK and the USA, respectively $[9,10]$. By contrast, in a case series conducted in Taiwan and Singapore over a period of 4-21 years, the prevalence of $K$. pneumoniae infection in endogenous endophthalmitis was found to be between 54 and $61 \%$ [2-4].

Certain capsular types of Klebsiella (K1 or K2) have been characterised as having a particular propensity to cause metastatic infection including endophthalmitis [11]. In 3 large 
Soon et al.: Klebsiella pneumoniae Infection: A Virulent Cause of Visual Loss

case series, $48-53 \%$ of cases with $K$. pneumoniae endophthalmitis were reported to have a liver abscess as a primary infection source [2-4]. Indeed, the incidence of endophthalmitis as a complication of a $K$. pneumoniae liver abscess is cited as being $7-11 \%[5,6]$. Over $50 \%$ of the affected patients also had a diagnosis of diabetes mellitus. In vitro, it has been shown that the neutrophils of diabetic patients have an impaired ability to phagocytose Klebsiella pneumoniae species with K1 and K2 surface antigens [11]. This impairment of host immunity may cause increased susceptibility of diabetic patients to K. pneumoniae endogenous endophthalmitis, and it also suggests that there may be clinical value in capsular phenotyping. The hypermucoviscous phenotype of $K$. pneumoniae and the magA and rmpA genes overlap with the $\mathrm{K} 1$ and $\mathrm{K} 2$ serotypes, and are also associated with invasive infection and increased inflammation $[12,13]$.

Visual outcomes following endogenous bacterial endophthalmitis are usually poor. A study of 22 consecutive patients by Yang et al. [5] found that $89 \%$ of patients achieved a visual outcome of light perception at best, with $41 \%$ requiring enucleation or evisceration. A case report by Ishii et al. [14] suggests that early vitrectomy and lensectomy may improve the outcome; however, the majority of cases are not identified until the disease is disseminated, and mortality from disseminated extra-ocular infection is 5\% [9]. Intravitreal antibiotics should be considered following intravitreal sampling in patients with suspected infectious endophthalmitis [15].

A study by Fung et al. [16] reported a $75 \%$ rate of faecal carriage of $K$. pneumoniae in healthy adults in Taiwan. This is greater than the 19.4-38\% reported in European studies. It is thought to be a predisposing factor for the higher rates of liver abscess in Asia. In our case, the patient had not visited South East Asia in 5 years, but she may have had enteric carriage. It has also been suggested that host genetic factors may play a role in determining susceptibility to Klebsiella infection, and studies in Drosophila have identified genes such as phg1 and kil1 essential for the intracellular killing of K. pneumoniae, which have known homologues in eukaryotes [17].

Klebsiella pneumoniae are commonly resistant to several classes of antibiotics and show an increasing prevalence of carbapenemase-producing strains worldwide. This presents a particular concern and makes choosing the right empirical treatment regimens difficult. Fortunately, our patient responded promptly to a third-generation cephalosporin.

\section{Conclusion}

This case report was unusual in that the primary source was initially occult, and the patient was a long-standing UK resident. It highlights known risk factors (Southeast Asian origin, diabetes mellitus and liver abscess) for K. pneumoniae infections. Any of these or a history of recent travel to Asia should prompt clinicians to consider this pathogen in a patient with endophthalmitis, even in the absence of positive cultures. K1 and K2 serotypes of Klebsiella spp. have been associated with more invasive infections. 16S ribotyping was shown to be a useful method to identify the organism to species level in this case.

\section{References}

Spadea L, Fiasca A: Treatment of post-operative endophthalmitis. Eur Ophthalmol Rev 2012;6:149-156. Wong JS, Chan TK, Lee HM, Chee SP: Endogenous bacterial endophthalmitis: an East Asian experience and a reappraisal of a severe ocular affliction. Ophthalmology 2000;107:1483-1491. 
Soon et al:: Klebsiella pneumoniae Infection: A Virulent Cause of Visual Loss

-3 Chen YJ, Kuo HK, Wu PC, Kuo ML, Tsai HH, Liu CC, Chen CH: A 10-year comparison of endogenous endophthalmitis outcomes: an east Asian experience with Klebsiella pneumoniae infection. Retina 2004;24:383-390.

4 Ang M, Jap A, Chee SP: Prognostic factors and outcomes in endogenous Klebsiella pneumoniae endophthalmitis. Am J Ophthalmol 2011;151:338-344.

-5 Yang CS, Tsai HY, Sung CS, Lin KH, Lee FL, Hsu WM: Endogenous Klebsiella endophthalmitis associated with pyogenic liver abscess. Ophthalmology 2007;114:876-880.

-6 Sheu SJ, Kung YH, Wu TT, Chang FP, Horng YH: Risk factors for endogenous endophthalmitis secondary to Klebsiella pneumoniae liver abscess: 20-year experience in Southern Taiwan. Retina 2011;31:2026-2031.

7 Abdul-Hamid A, Bailey SJ: Klebsiella pneumoniae liver abscess and endophthalmitis. BMJ Case Rep 2013, DOI: $10.1136 /$ bcr-2013-008690.

8 Durand ML: Endophthalmitis. Clin Microbiol Infect 2013;19:227-234.

-9 Okada AA, Johnson RP, Liles WC, D'Amico DJ, Baker AS: Endogenous bacterial endophthalmitis. Report of a ten-year retrospective study. Ophthalmology 1994;101:832-838.

10 Jackson TL, Eykyn SJ, Graham EM, Stanford MR: Endogenous bacterial endophthalmitis: a 17-year prospective series and review of 267 reported cases. Surv Ophthalmol 2003;48:403-423.

11 Lin JC, Siu LK, Fung CP, Tsou HH, Wang JJ, Chen CT, Wang SC, Chang FY: Impaired phagocytosis of capsular serotypes K1 or K2 Klebsiella pneumoniae in type 2 diabetes mellitus patients with poor glycemic control. J Clin Endocrinol Metab 2006;91:3084-3087.

12 Lee HC, Chuang YC, Yu WL, Lee NY, Chang CM, Ko NY, Wang LR, Ko WC: Clinical implications of hypermucoviscosity phenotype in Klebsiella pneumoniae isolates: association with invasive syndrome in patients with community-acquired bacteraemia. J Intern Med 2006;259:606-614.

13 Yeh KM, Lin JC, Yin FY, Fung CP, Hung HC, Siu LK, Chang FY: Revisiting the importance of virulence determinant magA and its surrounding genes in Klebsiella pneumoniae causing pyogenic liver abscesses: exact role in serotype K1 capsule formation. J Infect Dis 2010;201:1259-1267.

14 Ishii K, Hiraoka T, Kaji Y, Sakata N, Motoyama Y, Oshika T: Successful treatment of endogenous Klebsiella pneumoniae endophthalmitis: a case report. Int Ophthalmol 2011;31:29-31.

15 Radhika M, Mithal K, Bawdekar A, Dave V, Jindal A, Relhan N, Albini T, Pathengay A, Flynn H Jr: Pharmacokinetics of intravitreal antibiotics in endophthalmitis. J Ophthalmic Inflamm Infect 2014;4:22.

16 Fung CP, Lin YT, Lin JC, Chen TL, Yeh KM, Chang FY, Chuang HC, Wu HS, Tseng CP, Siu LK: Klebsiella pneumoniae in gastrointestinal tract and pyogenic abscess. Emerg Infect Dis 2012;18:1322-1325.

17 Benghezal M, Fauvarque MO, Tournebize R, et al: Specific host genes required for the killing of Klebsiella bacteria by phagocytes. Cell Microbiol 2006;8:139-148.

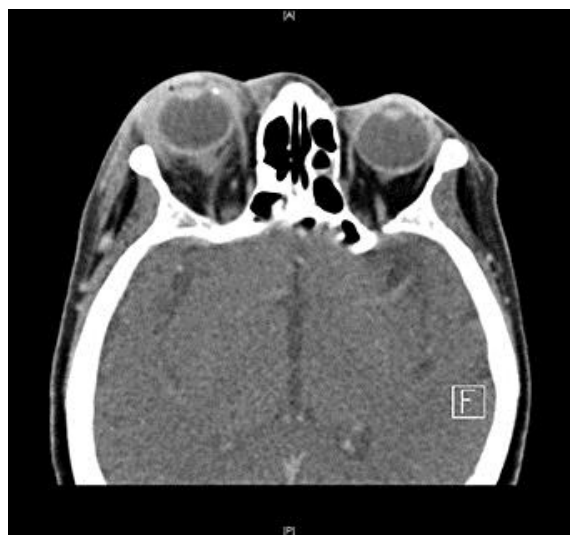

Fig. 1. Fat stranding and soft tissue swelling overlying the right globe anteriorly, which extends intra-conally and causes a mild degree of proptosis. 
Case Reports in

Ophthalmology

\begin{tabular}{l|l}
\hline \multicolumn{2}{l|}{ Case Rep Ophthalmol 2014;5:468-473 } \\
\hline DOI: $10.1159 / 000370145$ & $\begin{array}{l}\text { C 2014 S. Karger AG, Basel } \\
\text { www.karger.com/cop }\end{array}$ \\
\hline
\end{tabular}

Soon et al.: Klebsiella pneumoniae Infection: A Virulent Cause of Visual Loss
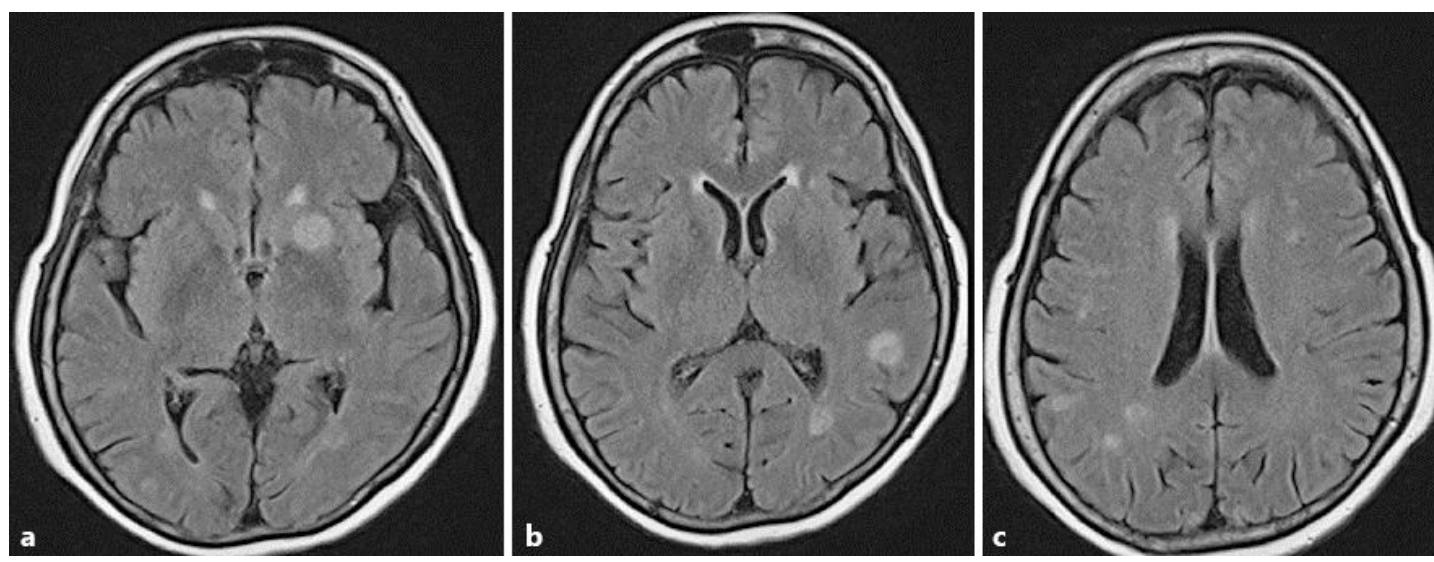

Fig. 2. a-c Multiple foci of tiny peripherally enhancing lesions demonstrating a slight T2-hypointense wall and surrounding oedema. The appearances are compatible with multiple microabscesses, most likely of a pyogenic origin.

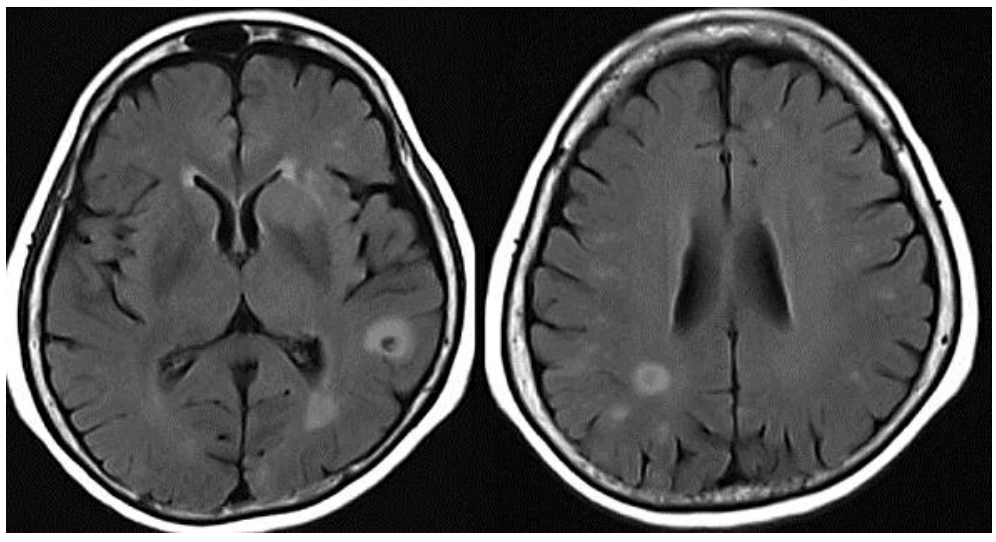

Fig. 3. A repeat MRI 3 days later showed progression in a perilesional oedema and a mild growth of the ring-enhancing lesions despite antibiotic therapy. 